\title{
Intra-individual plasticity of the $T A Z$ gene leading to different heritable mutations in siblings with Barth syndrome
}

\author{
Lorenzo Ferri ${ }^{1,2}$, Maria A Donati ${ }^{3}$, Silvia Funghini ${ }^{2}$, Catia Cavicchi ${ }^{2}$, Viviana Pensato ${ }^{4}$, Cinzia Gellera ${ }^{4}$, \\ Federica Natacci ${ }^{5}$, Luigina Spaccini ${ }^{6}$, Serena Gasperini ${ }^{7}$, Frédéric M Vaz ${ }^{8}$, David N Cooper ${ }^{9}$, Renzo Guerrini ${ }^{1,2}$ \\ and Amelia Morrone ${ }^{*, 1,2}$
}

Infantile-onset skeletal myopathy Barth syndrome (OMIM \#302060) is caused by mutations in the X-linked TAZ gene and hence usually manifests itself only in hemizygous males. Confirmatory testing is provided by mutational analysis of the $T A Z$ gene and/or by biochemical dosage of the monolysocardiolipin/tetralinoleoyl cardiolipin ratio. Heterozygous females do not usually display a clinical phenotype but may undergo molecular genetic prenatal diagnosis during pregnancy. We characterized two novel and non-identical TAZ gene rearrangements in the offspring of a single female carrier of Barth syndrome. The hg19chrX:g.153634427_153644361delinsKP_123427.1 TAZ gene rearrangement was identified in her affected son, whereas the NM_000116.3(TAZ)c. $-72 \_109+51$ del TAZ gene deletion was identified in a male foetus during a subsequent pregnancy. The unaffected mother was surprisingly found to harbour both variants in addition to a wild-type $T A Z$ allele. A combination of breakpoint junction sequencing, linkage analysis and assessment of allelic dosage revealed that the two variants had originated independently from an apparently unstable/mutable $T A Z$ maternal allele albeit via different mutational mechanisms. We conclude that molecular prenatal diagnosis in Barth syndrome families with probands carrying TAZ gene rearrangements should include investigation of the entire coding region of the $T A Z$ gene. The identification of the breakpoint junctions of such gross gene rearrangements is important to ensure accurate ascertainment of carriership with a view to providing appropriate genetic counselling.

European Journal of Human Genetics (2015) 23, 1708-1712; doi:10.1038/ejhg.2015.50; published online 18 March 2015

\section{INTRODUCTION}

Barth syndrome (BTHS; OMIM \#302060) is an X-linked, infantileonset inborn error of metabolism characterized by cardiomyopathy with or without left ventricular noncompaction, skeletal myopathy, hypotonia, growth delay and intermittent neutropenia. ${ }^{1-3} 3$-Methylglutaconic aciduria and lactic acidosis can represent early biochemical markers. ${ }^{1,4-6}$

The estimated prevalence of BTHS is around 1/300 000-400 000 live births (BSF, http://www.barthsyndrome.org/home), but evidence is growing that the disease may be underdiagnosed. ${ }^{2}$

BTHS is caused by mutations in the TAZ gene at Xq28, ${ }^{7}$ which encodes tafazzin, an acyltransferase which promotes molecular symmetry among cardiolipin (CL) species with different fatty acyl moieties. ${ }^{8,9} \mathrm{CL}$ is involved in the mitochondrial energy metabolism, mitochondrial dynamics and triggering of apoptotic pathways. ${ }^{10,11} \mathrm{In}$ mammalian cardiac and skeletal muscle, the predominant form is tetralinoleoyl cardiolipin (L4-CL). The impairment of tafazzin causes a L4-CL deficiency and an accumulation of intermediate species of monolysocardiolipins (MLCL) leading to profound ultrastructural and functional alterations of mitochondria. ${ }^{2,11-13}$
Confirmatory tests for the disease are both biochemical and molecular. Biochemical tests rely on the detection of an increased MLCL:L4-CL ratio in blood, tissues or cultured cell samples from patients. ${ }^{14,15}$ Molecular tests involve the mutational analysis of the $T A Z$ gene sequence, which should be considered mandatory as a few $T A Z$ gene mutations that do not lead to abnormal CL levels have recently been described. ${ }^{16}$

More than 100 different $T A Z$ gene mutations have been reported in the Human Tafazzin Gene Mutation and Variation Database of the American Barth Syndrome Foundation (http://www.barthsyndrome. org/science-medicine/human-tafazzin-(taz)-gene-mutation-variationdatabase) and $\sim 10 \%$ of these are gross $T A Z$ gene rearrangements. We have recently shown that the $T A Z$ gene sequence is enriched in interspersed repeats, sequence elements that can promote the formation of gross genetic rearrangements during meiosis or DNA replication. ${ }^{4,17}$

Here we describe the intriguing case of a BTHS family in which an asymptomatic mother gave birth to two male sons who harboured non-identical gross $T A Z$ gene rearrangements. If found not to be

${ }^{1}$ Department of Neurosciences, Pharmacology and Child Health, University of Florence, Florence, Italy; ${ }^{2}$ Paediatric Neurology Unit and Laboratories, Neuroscience Department, Meyer Children's Hospital, Florence, Italy; ${ }^{3}$ Metabolic and Muscular Unit, Neuroscience Department, Meyer Children's Hospital, Florence, Italy; ${ }^{4}$ Genetics of Neurodegenerative and Metabolic Diseases Unit, IRCCS Fondazione Istituto Neurologico Carlo Besta, Milan, Italy; ${ }^{5}$ Medical Genetics Unit, Ca' Granda IRCCS Foundation, Ospedale Maggiore Policlinico, Milan, Italy; ${ }^{6}$ Ambulatorio Genetica Medica, Ospedale 'Vittore Buzzi', Milan, Italy; ${ }^{7}$ Metabolic Unit, San Gerardo Hospital, Monza, Italy; ${ }^{8}$ Department of Clinical Chemistry and Paediatrics, Academic Medical Center, University of Amsterdam, Amsterdam, The Netherlands; ${ }^{9}$ Institute of Medical Genetics, School of Medicine, Cardiff University, Cardiff, UK

${ }^{*}$ Correspondence: Dr A Morrone, Paediatric Neurology Unit and Laboratories, Neuroscience Department, Meyer Children's Hospital, and Department of Neurosciences, Pharmacology and Child Health, University of Florence, viale Pieraccini 24, Florence, 50139, Italy. Tel: +39 0555662543; Fax: +39 0555662849; E-mail: a.morrone@meyer.it Received 7 August 2014; revised 6 February 2015; accepted 17 February 2015; published online 18 March 2015 
uncommon, such 'double hit' situations could complicate the molecular prenatal diagnosis of BTHS.

\section{MATERIALS AND METHODS}

\section{Family history}

The pedigree of the Italian BTHS family under study is presented in Figure 1. The proband (IV-L, Figure 1) was the first child born to non-consanguineous parents after a previous miscarriage. Owing to a family history of suspected $\mathrm{X}$-linked adrenoleukodystrophy (two deceased maternal uncles; II-C and II-D, Figure 1), the mother had elected to undergo prenatal diagnosis for increased very-long-chain fatty acid (VLCFA) levels in cultured amniocytes, but this proved normal. The child (IV-L) was born after 36 weeks gestation. After birth, he exhibited hypotonia and difficulty in suckling. In his first year of life, he manifested three severe single episodes of febrile seizures with ventricular tachycardia. Such manifestations occurred in association with otitis and gastroenteritis. Cardiological investigation revealed dilated cardiomyopathy with left ventricular noncompaction and ventricular arrhythmias. Further medical investigations revealed intermittent neutropenia and lactic acidosis. Anaemia was present and hypoglycaemia was intermittently detected. He started to walk at the age of 2 years although he exhibited reduced muscle tone and staggering. Muscle biopsy, performed as a consequence of the suspicion of a primary defect of the mitochondrial respiratory chain when the patient was 9 years, showed abnormal intracytoplasmic storage of lipids. At the age of 10 years, he came to our attention and BTHS was suspected due to the association of cardiomyopathy, neutropenia, 3-methylglutaconic aciduria and lactic acidosis.

We confirmed the diagnosis by monolysocardiolipin/cardiolipin analysis (MLCL/CL ratio on dried blood spot $^{14}$ was 5.51 ; normal $0-0.3$ ) and by $T A Z$ gene sequencing. At the time of writing, the patient is 11 years. He exhibits frequent episodes of debilitating headaches. Dilated cardiomyopathy and growth delay persist, with both his weight and height being under the 25th percentile.

A second son (IV-M, Figure 1) was born when IV-L was 5 years and before a definitive diagnosis had been made. Prenatal evaluation for VLCFA had been performed during pregnancy and was normal. Dilated cardiomyopathy was detected in IV-M on his second day of life. He also exhibited hypotonia, neutropenia and lactic acidosis. He died five and half months after birth due to sudden heart failure.

The mother (III-H in Figure 1) had a fourth pregnancy after the definitive diagnosis of BTHS had been made in the proband IV-L. She underwent genetic counselling and prenatal diagnosis for BTHS (IV-N, Figure 1).

\section{Mutational analysis of the $T A Z$ gene}

Isolation of genomic DNA and molecular analysis of the TAZ gene sequence were performed as previously described, ${ }^{4}$ after informed consent had been obtained from all subjects to be analysed, in accordance with local ethical committee recommendations. Multiplex PCR analyses were performed by using our previously reported primer pairs (Table 1 in Ferri et $a l^{4}$ ). PCR fragment combinations Taz3-4+Taz5+Taz6-7, Taz1-2+Taz8-9 and Taz1-2+Taz10-11 were successfully assayed.

\section{Long-range PCR analysis}

Long-range PCR (LR-PCR) using TaKaRa La Taq DNA polymerase (TaKaRa Bio Inc., Shiga, Japan) was performed to determine the precise locations of the breakpoint boundaries in proband IV-L. The primer pair used was DNASE1Lex10fw (5'-TGACCCTTCTAGCGTCCC- $\left.{ }^{\prime}\right)$ and Taz6-7rv (5'-AGGCCTAGTC TCAGCACCTG- $\left.3^{\prime}\right){ }^{4}$ The reverse primer Taz6-7rv anneals within intron 7 of $T A Z$, which was confirmed to be present in proband IV-L by routine amplification of the $T A Z$ coding region and intron-exon boundaries. Primer DNASE1L-ex10fw anneals $9.95 \mathrm{~kb}$ upstream of the start codon (ATG) of the $T A Z$ gene within exon 10 of the DNASE1L gene, which is located in the opposite orientation to, but partially overlaps with, the TAZ gene. The presence of this region in the patient's DNA was previously verified by PCR amplification and sequencing with the primer pair DNASE1L-ex10fw and DNASE1Lex10rv (5'-GATAGGACACTTGTGCAG-3'), which amplifies a $484 \mathrm{bp}$ fragment of DNASE1L exon 10. LR-PCR products were purified from a $0.8 \%$ agarose gel and sequenced by primer-walking. The Clustal W program (http:// www.clustal.org/) was used to align sequences located at the breakpoints. LR-PCR was also used to screen for heterozygous carriership of the hg19chrX:g.153634427_153644361delinsKP_123427.1 complex rearrangement.

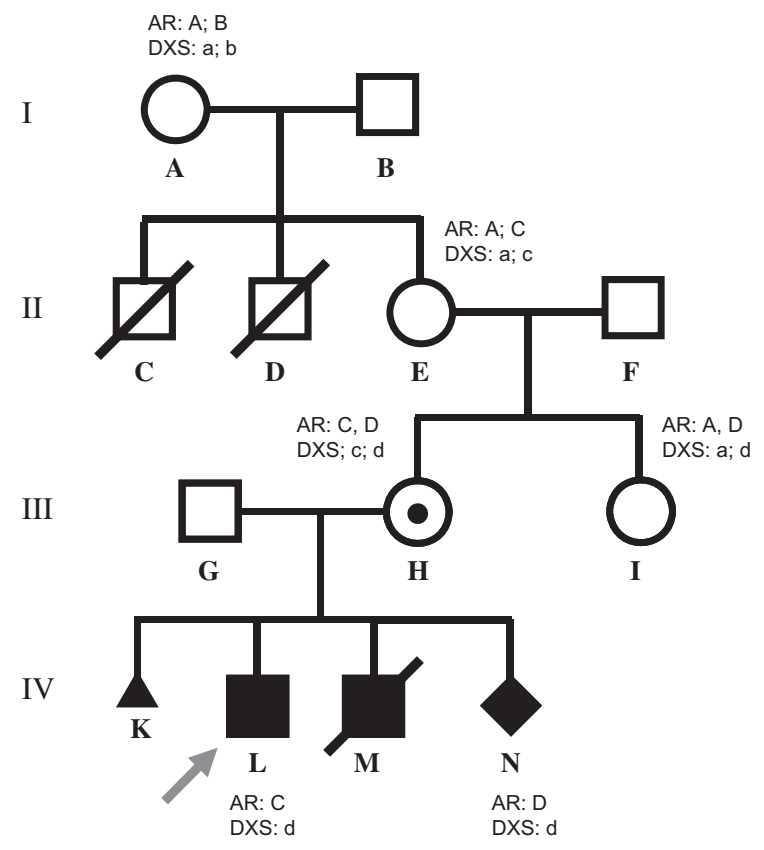

$A R$ (Androgen receptor gene)
$A=253$
$B=261$
$C=267$
$D=231$

DXS 8091 + DSX 1073

$\mathrm{a}=84 ; 312$

$b=90 ; 312$

$c=84 ; 310$

$d=72 ; 312$

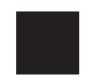

= Male with BTHS

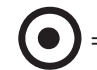

$=$ Female carrier of BTHS

$\Delta=$ Spontaneous abortion

Figure 1 Pedigree chart and results of the Xq linkage analysis. Linkage studies were performed in the family under study and involved analysis of DNA from individuals I-A, II-E, III-H, III-I, IV-L and IV-N. The polymorphic CAG repeats of the AR gene identified are indicated with upper case letters. The combination of loci DXS8091 and DXS1073 detected are denoted by lower case letters. 

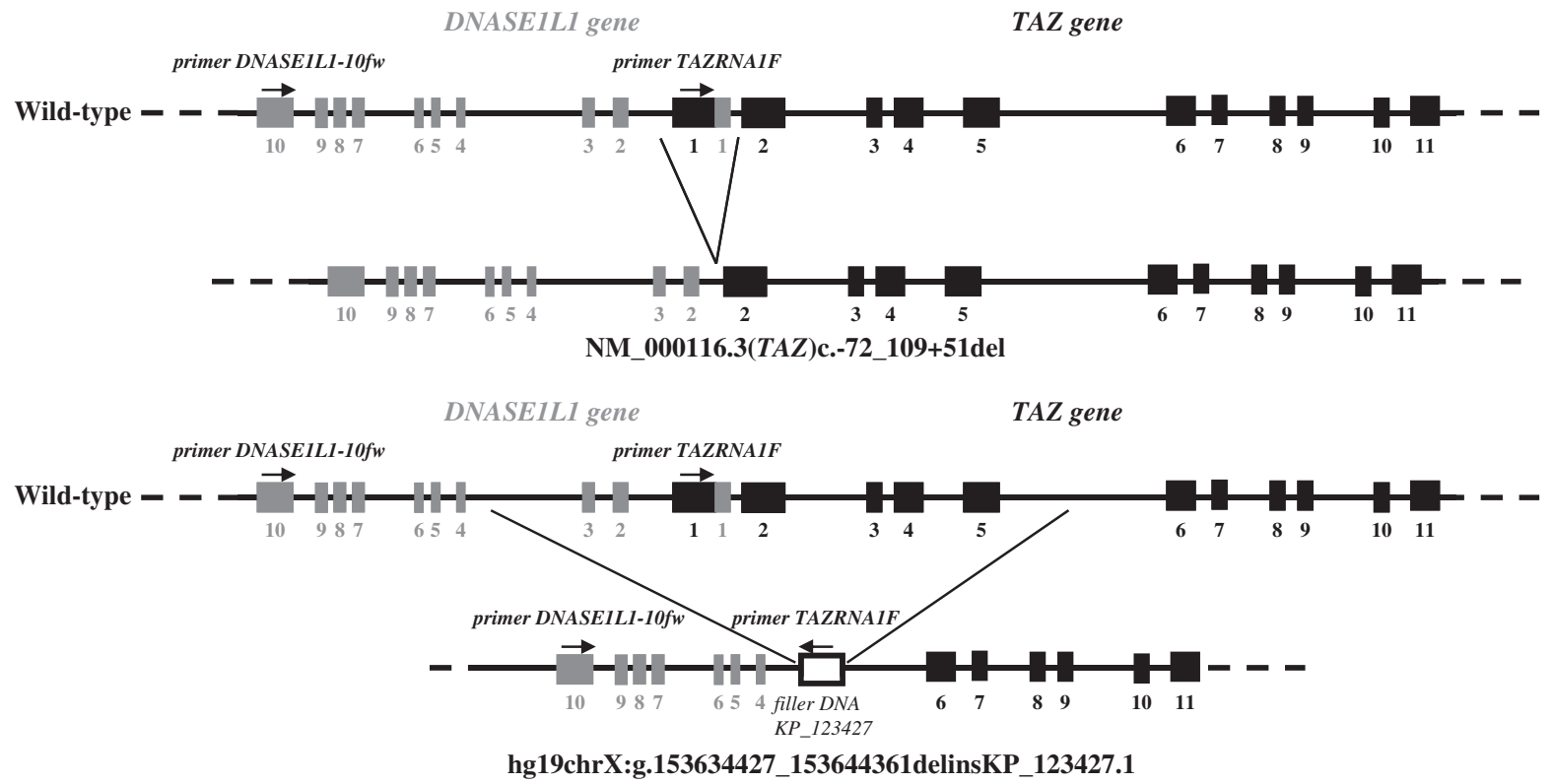

Figure 2 Representation of the TAZ gene rearrangements identified. Relative locations of the breakpoint junctions of NM_000116.3(TAZ)c. - 72_109+51del and hg19chrX:g.153634427_153644361delinsKP_123427.1. The annealing sites of primers DNASE1L-9fw and TAZRNA1F, which were used to confirm heterozygosity for the hg19chrX:g.153634427_153644361delinsKP_123427.1 variant in the proband's mother, are also indicated.

We combined the primer DNASE1L-ex10fw with primer TAZRNA1F (5'-ATGCCTCTGCACGTGAAGTG-3'), which anneals within TAZ exon 1 and is oriented as the primer DNASE1L-ex10fw in the wild-type TAZ allele (Figure 2). By contrast, in the hg19chrX:g.153634427_153644361delinsKP_ 123427.1 TAZ allele, although the annealing region of TAZRNA1F is present, it is in a reverse complement orientation. For this reason, the primer pair DNASE1L-ex10fw+TAZRNA1F amplifies specifically only the hg19chrX: g.153634427_153644361delinsKP_123427.1 TAZ allele (Figure 2). LR-PCR products obtained from the positive female III-H and the proband IV-L were purified from a $0.8 \%$ agarose gel and confirmed by DNA sequencing.

\section{Determination of the $\mathrm{X}$-chromosome linkage and $\mathrm{X}$-inactivation pattern}

The proximal region of Xq was tracked by the analysis of the polymorphic CAG repeats of the human androgen receptor gene $(A R ; \mathrm{Xq12})$ as previously reported. ${ }^{18}$ The distal Xq28 region carrying the TAZ gene was tracked instead by amplification of the two loci DXS8091 and DXS1073 from the ABI Prism Linkage Mapping Set (Applied Biosystems, Carlsbad, CA, USA) following the manufacturer's instructions. DXS8091 and DXS1073 both map to Xq28, upstream and downstream of $T A Z$, respectively.

The X-chromosome inactivation analysis was performed by studying the polymorphic CAG repeats of the $A R$ gene using the methylation-sensitive restriction enzymes $H p a I I$ and $C f o$ I as previously reported. ${ }^{18,19}$

\section{Quantitative fluorescent multiplex-PCR (QFM-PCR) of the TAZ gene}

TAZ exons 1, 4, 6-7, 8-9 and 10-11 were each amplified in a multiplex reaction, together with the ACY12 fragment from Ferri et $a l^{20}$ as an internal control peak. Primers used are detailed in Table 1.

Multiplex PCR reactions were performed in a $25 \mu \mathrm{l}$ final volume containing 1 U FastStart Taq DNA polymerase (Roche, Basel, Switzerland), $250 \mu \mathrm{M}$ each dNTP, 10 pmol each primer, $1 \times$ PCR buffer and $1.0 \mathrm{mM} \mathrm{MgCl}_{2}$. After primary denaturation for $5 \mathrm{~min}$ at $95^{\circ} \mathrm{C}$, amplification was carried out for 25 cycles of $30 \mathrm{~s}$ at $95^{\circ} \mathrm{C}, 30 \mathrm{~s}$ at $56^{\circ} \mathrm{C}$ and $30 \mathrm{~s}$ at $72^{\circ} \mathrm{C}$. PCR products were denatured for 5 min at $95^{\circ} \mathrm{C}$, run on an ABI PRISM 3130XL Genetic Analyser (Applied Biosystems) and sized using the GeneScan 500 LIZ Size Standard (Applied Biosystems) as the internal standard. For each sample, the peak areas of each
Table 1 Oligonucleotides used for QFM-PCR of TAZ gene

\begin{tabular}{lllc}
\hline Exon & Primer & Sequence $5^{\prime} \rightarrow 3^{\prime}$ & Length (bp) \\
\hline 1 & QF-TAZF-FAM & FAM-AGTCAGGGGCCAGTGTCTC & 207 \\
& QF-TAZR & GGTCCAGAAGCAGCTGTAGG & \\
4 & QF-TAZ4F-FAM & FAM-GCAGACATCTGCTTCACCAA & 159 \\
& TAZ 3-4R & CCATAGGTCCCTCCAAAACA & \\
6 6-7 & QF-TAZ6F-FAM & FAM-GGGTGCATATCTTCCCAGAA & 135 \\
& QF-TAZ7R & AAACGCAGGAATTCGGAAC & \\
$8-9$ & QF-TAZ8F-FAM & FAM-ATCTCAACCCCATCATCCTG & \multirow{2}{*}{186} \\
& QF-TAZ9R & TAGGGCGGACTGTTAGGAAG & \\
$10-11$ & QF-TAZ1OF-FAM & FAM-TTCAGTGCCCTGCCTGTACT & 229 \\
& QF-TAZ11R & GAGCTGCTCTGCCTGAGTCT & \\
\hline
\end{tabular}

TAZ fragment were normalized by dividing them by the peak area of the internal control ACY12. Normalized areas were then divided by the average normalized peak area from at least three normal controls. Values ranging from 0.7 to 1.3 were assumed to be normal based on our previous experience. ${ }^{20,21}$ Ten control DNAs and two DNAs from two previously described female relatives of a BTHS patient harbouring a heterozygous deletion of TAZ exons 6-11 (NG_009634.1:g.[9777_9814del; 9911-?_14402del] ${ }^{4}$ ) were included in order to validate the assay.

\section{Gene variants nomenclature and reference sequences}

Gene variants are given in the text by following the HGVS recommendations (http://www.hgvs.org/mutnomen/). Exon numbering refers to NG_009634.1 (TAZ; Gene ID: 6901) and NG_012884.1 (DNASE1L; Gene ID: 1774). GenBank reference sequences used for the description of gene variants are NM_000116.3 (TAZ cDNA) and NM_006730.2 (DNASE1L1 cDNA). The mutations identified have been submitted to the Human Tafazzin (TAZ) Gene Mutation and Variation Database, an official (and free access) database of $T A Z$ gene variants curated by the American Barth Syndrome Foundation (https://www.barthsyndrome.org/). 


\section{RESULTS}

The proband and foetus carry different $T A Z$ gene rearrangements and both variants are harboured by the mother

Single exon- and multiplex-PCR amplification of the TAZ-coding region revealed that proband IV-L harboured a mutated TAZ allele in which a portion of the gene encompassing exons $1-5$ was missing. We characterized the breakpoint junctions and identified a novel complex gene rearrangement of TAZ: hg19chrX:g.153634427_153644361 delinsKP_123427.1 [NM_006730.2(DNASE1L1)c.-517_NM_000116.3 (TAZ)c.461-3518delinsKP_123427]. The deleted region (9937 bp) included TAZ exons $1-5$ as well as exons $1-3$ of the DNASE1L1 gene. The $5^{\prime}$ deletion breakpoint is located within an AluY sequence (reverse orientation) while the $3^{\prime}$ breakpoint resides within an AluSx sequence (forward orientation). The breakpoint junctions included $706 \mathrm{bp}$ 'filler DNA' (KP_123427) comprising three distinct sequence subfragments (143 bp from NM_000116.3(TAZ)c. -82_c.+61, 527 bp from NM_000116.3(TAZ)c. $-640 \_c .-113$ and 36 bp from NM_000116.3 (TAZ)c.+73_c.+109), all derived from the deleted region. The 143 and $36 \mathrm{bp}$ fragments (Figure 2) both contained portions of the TAZ exon 1 -coding sequence and the $143 \mathrm{bp}$ fragment was present in reverse complement orientation. The $527 \mathrm{bp}$ fragment (Figure 2) contained part of the 5'-UTR of the TAZ gene.

Somewhat surprisingly, PCR amplification and sequencing analysis of the TAZ gene in foetal (IV-N) DNA (Figure 2) detected a different and novel NM_000116.3(TAZ)c. $-72 \_109+51$ del TAZ gross deletion that was quite distinct from the previously identified variant in the proband. The NM_000116.3(TAZ)c. $-72 \_109+51$ del variant serves to remove exon 1 of the TAZ gene and exon 1 of the flanking DNASE1L1 gene (Figure 2).

When we analysed the proband's mother's DNA (III-H, Figure 1), obtained from peripheral whole blood, we found that she harboured both variants, the NM_000116.3(TAZ)c. $-72 \_109+51$ del identical to that detected in her foetus IV-N and the hg19chrX: g.153634427_153644361delinsKP_123427.1, identical to that identified in the proband IV-L. Other female family members, III-I, II-E and I-A (Figure 1), were tested for both $T A Z$ rearrangements but none of them tested positive. Unfortunately, genotyping of BTHS patient IV-M was not possible, because he died before the family had received the diagnosis of BTHS and no biological material was available for postmortem DNA purification and analysis.

The two distinct mutations originated from rearrangements of the same $T A Z$ allele

To determine which $T A Z$ allele(s) had been rearranged to generate the $T A Z$ gene lesions identified, we performed a linkage analysis (Figure 1). The maternal Xq28 region was found to be shared by both males IV-L and IV-N, although they did not share the Xq proximal region harbouring the $A R$ gene. As the linkage between the $A R$ and DXS markers detected in the foetus IV-N is also evident in his aunt (III-I; Figure 1), the X-chromosome of IV-M must have arisen by crossing-over.

The X-inactivation studies performed on the proband's mother's DNA (III-H, Figure 1) detected an extremely skewed pattern (ratio >95:5) in favour of the wild-type allele (267 bp $A R$ fragment; Figure 1).

\section{Allelic dosage analysis of TAZ}

Data obtained from three replicates (Table 2) consistently failed to provide any evidence for allelic imbalance of the TAZ exons in the proband's mother's DNA. The efficacy of the method was, however, confirmed by the successful detection of heterozygosity of a TAZ exons 6-11 deletion in two DNAs from females harbouring the previously described NG_009634.1:g.[9777_9814del; 9911-?_14402del] TAZ complex rearrangement. ${ }^{4}$

\section{DISCUSSION}

The molecular diagnosis of monogenic inherited diseases is generally based on the detection of variants in the known disease gene of the probands. Once a nucleotide variant that affects the gene function has been detected, at-risk family members can be identified through a targeted search for the segregating familial mutation(s). The same principle pertains with regard to the prenatal molecular diagnosis of at-risk foetuses in the subsequent pregnancies of female carriers. It is in this context that we report the unusual case of a family in which a clinically unaffected mother harboured two novel and quite different $T A Z$ gene rearrangements that were independently inherited by her male offspring (one child and one foetus).

Analysis of the breakpoint junctions of the two novel rearrangements were consistent with the view that they originated via different mutational mechanisms. The NM_000116.3(TAZ)c. -72_109+51del occurred between two GC-rich regions, possibly through replication slippage. $^{22}$ By contrast, the hg19chrX:g.153634427_153644361 delinsKP_123427.1 complex allele does not exhibit significant sequence homology between the breakpoint junctions, suggesting that this rearrangement could have been mediated by double-strand breaks repaired by addition of a DNA stuffer derived from portions of the deleted fragment.

By means of a linkage analysis of the $\mathrm{Xq}$ region within the involved family members, we ascertained that the NM_000116.3(TAZ) c. -72_109+51del and hg19chrX:g.153634427_153644361delinsKP_ 123427.1 rearrangements had originated from the same apparently unstable/mutable maternal TAZ allele. The proband's maternal lineage was also tested, but no other individual (including his grandmother, great grandmother or maternal aunt) harboured any of the identified $T A Z$ variants.

The coexistence of two different $T A Z$ variants in the genome of the unaffected mother could be explicable in terms of somatic and gonadal mosaicism. Alternatively, each maternal cell might carry both variants together with the wild-type $T A Z$ allele. This second hypothesis

Table 2 QFM-PCR results

Fluorescence ratios ${ }^{\mathrm{a}}$

\begin{tabular}{|c|c|c|c|c|c|}
\hline \multirow[b]{2}{*}{ Patient } & \\
\hline & Exon 1 & Exon 4 & Exons 6-7 & Exons 8-9 & Exons $10-11$ \\
\hline $\mathrm{III}-\mathrm{H}$ & 0.82 & 1.15 & 1.11 & 0.93 & 1.05 \\
\hline Heterozygous $\triangle T A Z$ 6-11 (female 1$)^{\mathrm{b}}$ & 1.07 & 0.95 & 0.62 & 0.63 & 0.60 \\
\hline Heterozygous $\triangle T A Z$ 6-11 (female 2$)^{\mathrm{b}}$ & 1.12 & 1.00 & 0.63 & 0.61 & 0.62 \\
\hline
\end{tabular}

aFluorescence ratios are calculated as indicated in the Materials and Methods section and are the average of two experiments.

bFemales harbouring the NG_009634.1:g.[9777_9814del; 9911-?_14402del] TAZ complex rearrangement [4]; see Materials and Methods section. 
is inherently unlikely as the male proband and the male foetus were positive for only one $T A Z$ gene rearrangement and did not harbour a normal $T A Z$ allele. Further, under this second hypothesis, an allelic imbalance of $T A Z$ exons 6-11 would be expected in the mother's DNA. As the analysis of allelic dosage that we performed did not reveal any clear-cut imbalance of $T A Z$ exons 6-11 in the mother, somatic and gonadal mosaicism appears to be the more likely explanation in this particular case.

Other examples of co-occurring pathological mutations in relatives have been reported for other X-linked diseases such as Duchenne/ Becker muscular dystrophy ${ }^{23-27}$ and haemophilia A. ${ }^{28,29}$ Similar cases in autosomal genes have also been reported, for example, involving the NF1 gene, ${ }^{30,31}$ the RB1 gene ${ }^{32}$ and the APC gene. ${ }^{33}$ It remains unclear whether these cases represent examples of mutationally unstable alleles or simply chance co-occurrence.

Both the identified $T A Z$ variants involve deletions of portions of the deoxyribonuclease I-like 1 gene (DNASE1L1), which partially overlaps with TAZ. A similar case has been previously described in a BTHS patient who harboured a deletion of the entire $T A Z$ gene and a partial deletion of DNASE1L. ${ }^{34}$ However, no disease has so far been associated with mutation of the DNASE1L1 gene and therefore there is no evidence for any involvement of this gene in the BTHS clinical phenotype. ${ }^{34}$

Our observations should be borne in mind when performing molecular diagnosis of BTHS. For instance, when performing molecular prenatal diagnosis of BTHS in families with large TAZ gene rearrangements, we recommend investigating the entire coding region of the TAZ gene by both multiplex PCR amplification of all exons and by direct sequencing. Moreover, additional efforts should be made to develop rapid and simple molecular tools to identify gross genetic rearrangements of the TAZ gene in heterozygous females (eg, MLPA or Real-Time PCR-based assay).

\section{CONFLICT OF INTEREST}

The authors declare no conflict of interest.

\section{ACKNOWLEDGEMENTS}

The Fondazione Meyer ONLUS, Firenze, Italia and the Regione Toscana, Italia are gratefully acknowledged for their financial support (grant 'POR CRO FSE 2007-2013, MEYER AON-CHAP'). This work was also partially supported by AMMeC (Associazione Malattie Metaboliche Congenite, Italia). We express our gratitude to the Barth Italia Foundation and to the affected family whose collaboration made this study possible.

1 Barth PG, Scholte HR, Berden JA et al: An X-linked mitochondrial disease affecting cardiac muscle, skeletal muscle and neutrophil leucocytes. J Neurol Sci 1983; 62: 327-355.

2 Clarke SL, Bowron A, Gonzalez IL et al: Barth syndrome. Orphanet J Rare Dis 2013; 8: 23.

3 Spencer CT, Bryant RM, Day J et al: Cardiac and clinical phenotype in Barth syndrome. Pediatrics 2006; 118: e337-e346.

4 Ferri L, Donati MA, Funghini S et al: New clinical and molecular insights on Barth syndrome. Orphanet J Rare Dis 2013; 8: 27.

5 Kelley RI, Cheatham JP, Clark BJ et al: X-linked dilated cardiomyopathy with neutropenia, growth retardation, and 3-methylglutaconic aciduria. J Pediatr 1991; 119: 738-747.

6 Steward CG, Newbury-Ecob RA, Hastings R et al: Barth syndrome: an X-linked cause of fetal cardiomyopathy and stillbirth. Prenat Diagn 2010; 30: 970-976.
7 Bione S, D'Adamo P, Maestrini E, Gedeon AK, Bolhuis PA, Toniolo D: A novel X-linked gene, G4.5. is responsible for Barth syndrome. Nat Genet 1996; 12: 385-389.

8 Houtkooper RH, Turkenburg M, Poll-The BT et al: The enigmatic role of tafazzin in cardiolipin metabolism. Biochim Biophys Acta 2009; 1788: 2003-2014.

9 Malhotra A, Xu Y, Ren M, Schlame M: Formation of molecular species of mitochondrial cardiolipin. 1. A novel transacylation mechanism to shuttle fatty acids between sn-1 and sn-2 positions of multiple phospholipid species. Biochim Biophys Acta 2009; 1791: 314-320.

10 Gonzalvez F, Gottlieb E: Cardiolipin: setting the beat of apoptosis. Apoptosis 2007; 12: 877-885.

11 Schlame M, Rua D, Greenberg ML: The biosynthesis and functional role of cardiolipin. Prog Lipid Res 2000; 39: 257-288.

12 Schlame M, Towbin JA, Heerdt PM, Jehle R, DiMauro S, Blanck TJ: Deficiency of tetralinoleoyl-cardiolipin in Barth syndrome. Ann Neurol 2002; 51: 634-637.

13 Valianpour F, Mitsakos V, Schlemmer D et al: Monolysocardiolipins accumulate in Barth syndrome but do not lead to enhanced apoptosis. J Lipid Res 2005; 46: 1182-1195.

14 Houtkooper RH, Rodenburg RJ, Thiels $\mathrm{C}$ et al: Cardiolipin and monolysocardiolipin analysis in fibroblasts, lymphocytes, and tissues using high-performance liquid chromatography-mass spectrometry as a diagnostic test for Barth syndrome. Anal Biochem 2009; 387: 230-237.

15 Kulik W, van Lenthe H, Stet FS et al: Bloodspot assay using HPLC-tandem mass spectrometry for detection of Barth syndrome. Clin Chem 2008; 54: 371-378.

16 Bowron $\mathrm{A}$, Honeychurch J, Williams $\mathrm{M}$ et al: Barth syndrome without tetralinoleoyl cardiolipin deficiency: a possible ameliorated phenotype. J Inher Metab Dis 2015; 38: 279-286.

17 Boone PM, Yuan B, Campbell IM et al: The Alu-rich genomic architecture of SPAST predisposes to diverse and functionally distinct disease-associated CNV alleles. Am J Hum Genet 2014; 95: 143-161.

18 Pegoraro E, Schimke RN, Arahata K et al: Detection of new paternal dystrophin gene mutations in isolated cases of dystrophinopathy in females. Am J Hum Genet 1994 54: 989-1003.

19 Morrone A, Cavicchi C, Bardelli T et al: Fabry disease: molecular studies in Italian patients and X inactivation analysis in manifesting carriers. J Med Genet 2003; 40: e103.

20 Ferri L, Funghini S, Fioravanti A et al: Aminoacylase I deficiency due to ACY1 mRNA exon skipping. Clin Genet 2014; 86: 367-372

21 Ferri L, Caciotti A, Cavicchi $\mathrm{C}$ et al: Integration of PCR-sequencing analysis with multiplex ligation-dependent probe amplification for diagnosis of hereditary fructose intolerance. JIMD Rep 2012; 6: 31-37.

22 Gu W, Zhang F, Lupski JR: Mechanisms for human genomic rearrangements. Pathogenetics 2008; 1: 4.

23 Laing NG, Layton MG, Johnsen RD et al: Two distinct mutations in a single dystrophin gene: chance occurrence or premutation? Am J Med Genet 1992; 42: 688-692.

24 Miciak A, Keen A, Jadayel D, Bundey S: Multiple mutation in an extended Duchenne muscular dystrophy family. J Med Genet 1992; 29: 123-126.

25 Morandi L, Mora M, Tedeschi S et al: DMD and BMD in the same family due to distinct mutations. Am J Med Genet 1995; 59: 501-505.

26 Mostacciuolo ML, Miorin M, Vitiello L et al: Occurrence of two different intragenic deletions in two male relatives affected with Duchenne muscular dystrophy. Am J Med Genet 1994; 50: 84-86

27 Zatz M, Sumita D, Campiotto S et al: Paternal inheritance or different mutations in maternally related patients occur in about $3 \%$ of Duchenne familial cases. Am J Med Genet 1998; 78: 361-365.

28 Gitschier J: Maternal duplication associated with gene deletion in sporadic hemophilia. Am J Hum Genet 1988; 43: 274-279.

29 Tizzano EF, Vencesla A, Baena M et al: First report of two independent point factor VIII mutations in a family with haemophilia A: a word of caution for carrier diagnosis. Thromb Haemost 2005; 94: 675-677.

30 Klose A, Peters $\mathrm{H}$, Hoffmeyer $\mathrm{S}$ et al: Two independent mutations in a family with neurofibromatosis type 1 (NF1). Am J Med Genet 1999; 83: 6-12.

31 Upadhyaya M, Majounie E, Thompson P et al: Three different pathological lesions in the NF1 gene originating de novo in a family with neurofibromatosis type 1. Hum Genet 2003; 112: 12-17.

32 Munier FL, Wang MX, Spence MA et al: Pseudo low penetrance in retinoblastoma. Fortuitous familial aggregation of sporadic cases caused by independently derived mutations in two large pedigrees. Arch Ophthalmol 1993; 111: 1507-1511.

33 Davidson S, Leshanski L, Rennert G, Eidelman S, Amikam D: Maternal mosaicism for a second mutational event-a novel deletion-in a familial adenomatous polyposis family harboring a new germ-line mutation in the alternatively spliced-exon 9 region of APC. Hum Mutat 2002; 19: 83-84.

34 Singh HR, Yang Z, Siddiqui S et al: A novel Alu-mediated Xq28 microdeletion ablates $T A Z$ and partially deletes $D N L 1 L$ in a patient with Barth syndrome. Am J Med Genet $A$ 2009; 149A: 1082-1085. 